\title{
Madrid at the center of the Spanish COVID-19 pandemic: the result of ill-advised political decision-making
}

\author{
Alberto Ortiz ${ }^{1}$ iD \\ ${ }^{1}$ IIS-Fundación Jiménez Díaz UAM and School of Medicine, UAM, Madrid, Spain
}

\section{ABSTRACT}

In this issue of the Portuguese Journal of Nephrology and Hypertension, Deira et al report on the epidemiology of coronavirus disease 2019 (COVID-19) in Spanish dialysis units outside the largest cities. This unique viewpoint allows to further trace the spread of the pandemic from Madrid, which became the epicenter of COVID-19 in Spain as a consequence of successive ill-advised political decisions. Deira et al further observed a disconnect between general population infection rates and dialysis unit infection rates in several provinces, pointing to local factors that should be identified and corrected prior to the next pandemic. Although they did not provide specific data on the topic, prior studies from Spain characterized shared healthcare transport to and for dialysis units as a key contributor to infection rates among hemodialysis patients.

Key-words: hemodialysis, COVID-19, epidemiology, Madrid, Spain

(C) 2021 Portuguese Journal of Nephrology \& Hypertension. Published by Publicações Ciência \& Vida This is an open access article under the CC BY-NC-ND license (http://creativecommons.org/licenses/by-nc-nd/4.0/).

Chronic kidney disease (CKD) represents the most common global risk factor for severe coronavirus disease 2019 (COVID-19), after older age $^{1}$. It also represents the risk factor that most increases the risk of death in COVID-19 patients, again, after older age $\mathrm{e}^{1}$. The risk of death from COVID-19 is highest for dialysis patients and for patients with functioning kidney grafts, but it is already increased persons with CKD category $\mathrm{G} 5$, i.e. in patients with estimated glomerular filtration rate 30 to $60 \mathrm{ml} / \mathrm{min} / 1.73 \mathrm{~m}^{2} 2$ (Figure 1). The very high risk of severe COVID-19 of dialysis patients may have been predicted from their increased susceptibility to death from other infections ${ }^{3}$, although a detailed exploration of the cellular and molecular mechanisms responsible for the increased risk may be useful to develop novel strategies that protect persons with CKD from future pandemics. Additionally, one of the multiple failures of the Spanish healthcare system regarding preparedness for the COVID-19 pandemic disproportionality affected dialysis patients: dialysis patients continued to use shared rides to access dialysis units thrice weekly, at a time when the Spanish COVID-19 czar, Fernando Simon, was actively discouraging the use of face masks to prevent severe acute respiratory syndrome coronavirus-2 (SARS-CoV-2) transmission ${ }^{4}$. As a result, transport to and from dialysis units became a key risk factor for SARS-CoV-2 infection among hemodialysis patients ${ }^{5}$. Additionally, the lack of appropriate personal protective equipment contributed to SARS-CoV-2 infection of nephrologists, several of whom died during the pandemic, including Professor Alberto Tejedor, and other healthcare personnel ${ }^{6}$. The lack of appropriate personal protective equipment may have also contributed to spread SARS-CoV-2 to kidney disease patients, especially in the early stages of the pandemic.

\section{Figure 1}

The contribution of governments to the expansion of the COVID-19 pandemic outside China. Deaths per 100000 as of 6 May 2020. Source: reference 4.

Some Madrid hospitals had already collapsed under COVID-19
by March 6 , but the Spanish Government led a massive
demonstration on March 8 in central Madrid. Several participating
ministers infected. Mortality explodes in the next few weeks
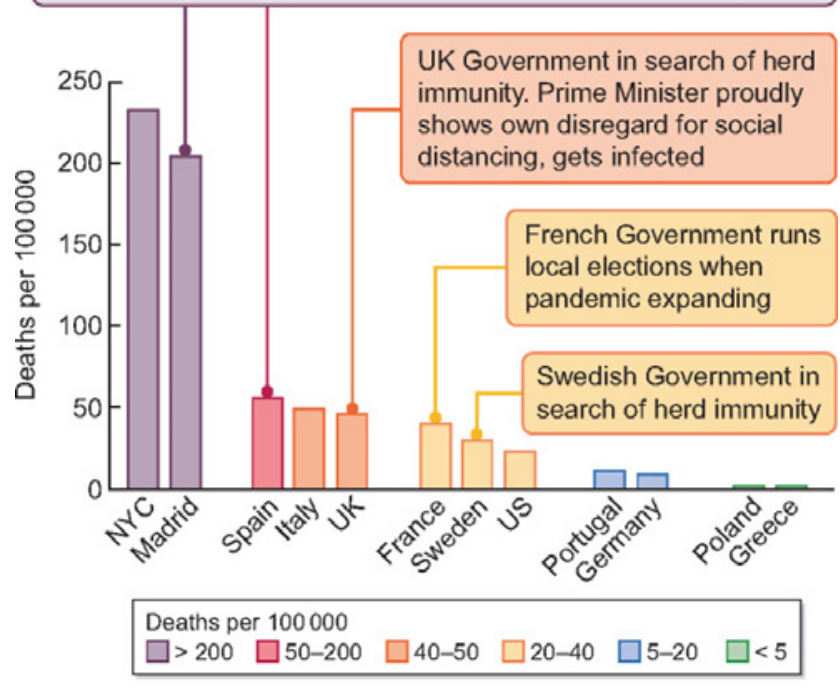
Deira et al now report on COVID-19 incidence in hemodialysis units from less populated regions of Spain, outside the large centers in Madrid and Barcelona ${ }^{7}$. This is a welcome addition to information on the impact of the pandemic on hemodialysis patients in Spain that up to now had been derived from or included mainly Madrid and Barcelona data. A key observation is that the incidence of COVID-19 was inversely related to the distance from Madrid. Indeed, Madrid was the epicenter of the COVID-19 pandemic in Spain. A series of ill-fated decisions by health authorities may have contributed to the disaster. Limitations on SARS-CoV-2 testing were imposed that contributed to the unnoticed spread of the disease: only patients with history of travel to China and, later, to Italy, were allowed to be tested. There were no limitations on travel to and from highly affected areas of China or Italy. And no real preparation had been made for the pandemic from the point of view of personal protective equipment or of setting up diagnostic capacity or trace capabilities. By March 6, 2021, some smaller hospitals in the Madrid periphery were already overwhelmed by COVID-19 patients. Patients needing both general hospitalization and Intensive Care hospitalization had to be transferred to larger hospitals. At my center, the Fundación Jiménez Díaz University Hospital, we received such patients on Friday 6 and Saturday 7 March 2020. Despite this obviously serious situation, massive gatherings expected for that weekend went ahead as planned. More surprisingly, several ministers in the Spanish government actively promoted the participation in a mass gathering in Madrid in the evening of March 8, 2021. In 2019, over 500000 persons attended. Day after day, government ministers appeared on TV, downplaying the pandemic and promoting attendance at the mass gathering, up to the same day of the gathering. Perhaps the high point of this terrible mistake took place when Fernando Simon indicated that he would not prevent his children from attending the gathering. As explained above, at this point, he was actively discouraging the use of face masks to prevent transmission of the disease. Fortunately, the population of Madrid appeared to be wiser than the Spanish government and "only" 130 000 persons attended. Nevertheless, there is evidence that this massive gathering at a time when the pandemic was taking off may have represented a massive superspreader event. Thus, $40 \%$ of attending government ministers were infected by SARS-CoV-2 within the expected incubation period of the virus and one of them, who had actively and publicly downplayed the dangers posed by COVID-19, needed hospitalization. The rest of the story is well known. The government that had denied the seriousness of the pandemic on March 8 had to close the country on March 14. Despite this, the number of hospitalizations and deaths in Madrid soared over the next two weeks. The health system was overwhelmed by the sheer number of persons with severe COVID-19. There were no resources to care for all. At one point, $90 \%$ of inpatients at my center were COVID-19 patients. Persons died at home and at hospices without proper care. There were not enough ventilators nor acute dialysis facilities for all that developed respiratory failure or acute kidney injury requiring replacement of organ function. Bodies accumulated in large storage facilities. Official COVID-19 deaths underestimated the real excess mortality from COVID-19 since diagnostic capacity was well below the needs for so many patients. I spent 2 weeks of daily fever at home with COVID-19, without any treatment beyond acetaminophen, and visited the emergency room twice, as a patient, when oxygen saturation, that I was monitoring myself, fell below 93\%. Bilateral pneumonia was evident in the chest X-ray, but hospitalization was reserved for persons with more severe disease. As pointed out by Deira et al, the situation was very different outside Madrid. Even in the Basque country and Barcelona, other hard-hit areas, the situation was not as dramatic. Now Deira et al provide evidence on how the pandemic expanding from Madrid affected dialysis patients in other parts of Spain.

A second observation by Deira et al is that the incidence of COVID-19 in individual dialysis units was not closely related to the local incidence of the disease in the general population? ${ }^{7}$. There may be several potential explanations for this observation, including the role of chance and availability of personal protective equipment in individual units. This latter point is not discussed by Deira et al. However, it may have contributed to spreading the virus within hospitals, in which infection was more common that in self-standing dialysis units. The role and impact of transport to and from the dialysis units is also not discussed. This may have magnified the impact of COVID-19 on individual units, if one infected patient shared the ride with several others, in the absence of face masks. These rides may take up to 45-60 minutes in a closed environment, enough to infect other persons in the same vehicle.

In conclusion, reporting experiences from dialysis centers from different cities and environments is key to defining the full extent of the pandemic in dialysis patients, to provide a balanced view and to learn from past mistakes, as globalization, increasing human populations impinging on natural habitats, and global warming are expected to increase the risk and speed of spread of future pandemics.

Disclosure of potential conflicts of interest: $\mathrm{AO}$ has received consultancy or speaker fees or travel support from Astellas, Astrazeneca, Amicus, Amgen, Fresenius Medical Care, Bayer, Sanofi-Genzyme, Menarini, Kyowa Kirin, Alexion, Otsuka and Vifor Fresenius Medical Care Renal Pharma and is Director of the Catedra Mundipharma-UAM of diabetic kidney disease and the Catedra Astrazeneca-UAM of chronic kidney disease and electrolytes.

\section{References}

1. ERA-EDTA Council; ERACODA Working Group. Chronic kidney disease is a key risk factor for severe COVID-19: a call to action by the ERA-EDTA. Nephrol Dial Transplant. 2021:36(1):87-94.

2. Perez-Gomez MV, Bartsch LA, Castillo-Rodriguez E, et al. Clarifying the concept of chronic kidney disease for non-nephrologists. Clin Kidney J. 2019;12(2):258-261.

3. Ortiz A, Covic A, Fliser D, et al. Epidemiology, contributors to, and clinical trials of mortality risk in chronic kidney failure. Lancet. 2014;383(9931):1831-184.

4. Carriazo S, Kanbay M, Ortiz A. Kidney disease and electrolytes in COVID-19: more than meets the eye. Clin Kidney J. 2020;13(3):274-280.

5. Fernandez-Prado R, Gonzalez-Parra E, Ortiz A. Often forgotten, transport modality to dialysis may be life-saving. Clin Kidney J. 2020;13(4):510-512.

6. Quiroga B, Sánchez-Álvarez E, Ortiz A, de Sequera P; Spanish Society of Nephrology. Suboptimal personal protective equipment and SARS-CoV-2 infection in Nephrologists: a Spanish national survey. Clin Kidney J. 2021;14(4):1216-1221.

7. Deira JL et al. Covid-19 in 40 Dialysis Facilities. A prospective multicenter cohort study in Spain. Portuguese Journal of Nephrology and Hypertension 2021 (this issue).

\section{ORCID}

Alberto Ortiz (iD) 0000-0002-9805-9523

\section{Correspondence to:}

Alberto Ortiz

IIS-Fundacion Jimenez Diaz, Av Reyes Católicos 2, 28040, Madrid, Spain

E-mail: aortiz@fjd.es 